\title{
Raman thermometry based thermal conductivity measurement of bovine cortical bone as a function of compressive stress
}

\author{
Zhang, Yang; Tomar, Vikas, Purdue University, West Lafayette, United States
}

\begin{abstract}
Biological materials such as bone have microstructure that incorporates a presence of a significant number of interfaces in a hierarchical manner that lead to a unique combination of properties such as toughness and hardness. However, studies regarding the influence of structural hierarchy on physical properties such as thermal conductivity and its correlation with mechanical stress of biomaterials are limited. Such studies can point out important insights regarding the role of biological structural hierarchy in influencing mechanophysical properties. This study presents an analytic-experimental approach to establish stress-thermal conductivity correlation in bovine cortical bone as a function of nanomechanical compressive stress using Raman thermometry. Analyses establish empirical relations between Raman shift and temperature as well as a relation between Raman shift and nanomechanical compressive stress. Analyses verify earlier reported thermal conductivity results at $0 \%$ strain and room temperature. In addition, measured trends and established thermal conductivity-stress relation indicates that the thermal conductivity values increases and then decrease as a function of increase in compressive strain.
\end{abstract}

